\title{
Time Operator in Quantum Mechanics
}

\author{
Adwit Kanti Routh \\ Department of Physics, National University Bangladesh, Gazipur, Bangladesh \\ Email: adwitkantirouth7@gmail.com
}

How to cite this paper: Routh, A.K. (2019) Time Operator in Quantum Mechanics. Open Access Library Journal, 6: e5816. https://doi.org/10.4236/oalib.1105816

Received: September 24, 2019

Accepted: November 5, 2019

Published: November 8, 2019

Copyright () 2019 by author(s) and Open Access Library Inc.

This work is licensed under the Creative Commons Attribution International License (CC BY 4.0).

http://creativecommons.org/licenses/by/4.0/

\begin{abstract}
We can not only bring time operator in quantum mechanics (non-relativistic) but also determine its Eigen value, commutation relation of its square with energy and some of the properties of time operator like either it is Hermitian or not, either its expectation value is real or complex for a wave packet etc. Exactly these are what I have done.
\end{abstract}

\section{Subject Areas}

Quantum Mechanics

\section{Keywords}

Quantum Mechanics, Quantum Physics, Quantum Operators, Time Operators

\section{Introduction}

Any kind of measurement is organized in space \& time. Normally physical quantities are either function of space or function of time and sometimes function of both. In non-relativistic quantum mechanics, though wave function depends on both space and time, only space enters quantum mechanics as operator, time does not. There is no time operator in quantum mechanics. All quantum mechanics textbooks introduce only space as operator, not time. But it is not only possible to produce time operator in quantum mechanics but also we can find out Eigen value of time operator, determines its square's commutation relation with energy, and prove that it is Hermitian operator; the expectation value of time operator is real for a wave packet and any two wave functions $\Psi(r, t)$ and $\phi(r, E)$ are Fourier transform of each other, where $r, t, E$ represents respectively position, time and energy of any quantum mechanical particle.

\section{Origin of Time Operator}

If wave nature of some quantum mechanical free particle is described by de 
Broglie wave function [1]

$$
\Psi(\boldsymbol{r}, t)=A \mathrm{e}^{i(\boldsymbol{k} \cdot \boldsymbol{r}-\omega t)}
$$

where, $\boldsymbol{k}$ is wave number, $\omega$ is angular frequency.

$$
\begin{gathered}
\left.\Rightarrow \Psi(\boldsymbol{r}, t)=A \mathrm{e}^{i \frac{(\boldsymbol{p} \cdot \boldsymbol{r}-E t)}{\hbar}} \quad \text { [Momentum } \boldsymbol{p}=\hbar \boldsymbol{k}, E=\hbar \omega\right] \\
\Rightarrow \frac{\partial \Psi(\boldsymbol{r}, t)}{\partial E}=-\frac{i}{\hbar} t A \mathrm{e}^{i \frac{(\boldsymbol{p} \cdot \boldsymbol{r}-E t)}{\hbar}} \\
\Rightarrow \frac{\partial \Psi(\boldsymbol{r}, t)}{\partial E}=-\frac{i}{\hbar} t \Psi(\boldsymbol{r}, t) \\
\Rightarrow \frac{\partial}{\partial E}=-\frac{i}{\hbar} t \\
\Rightarrow \hat{t}=-\frac{\hbar}{i} \frac{\partial}{\partial E} \\
\Rightarrow \hat{t}=i^{2} \frac{\hbar}{i} \frac{\partial}{\partial E} \\
\Rightarrow \hat{t}=i \hbar \frac{\partial}{\partial E}
\end{gathered}
$$

This is time operator. Though in Zhi-Yong Wang's paper [2] there was a minus sign in front of " $i \hbar \frac{\partial}{\partial E}$ ”. We can omit that.

\section{Eigen Value of Time Operator}

Now we will see how to find out its Eigen value. We have known,

$$
\begin{gathered}
\hat{t}=i \hbar \frac{\partial}{\partial E} \\
\Rightarrow \hat{t} \Psi=i \hbar \frac{\partial}{\partial E} \Psi \\
\Rightarrow \hat{t} \Psi=i \hbar \frac{\partial}{\partial E}\left(A \mathrm{e}^{\left.i \frac{(p \cdot r-E t)}{\hbar}\right)}\right) \\
\Rightarrow \hat{t} \Psi=i \hbar A\left(-\frac{i}{\hbar} t\right) \mathrm{e}^{-i E t} \\
\Rightarrow \hat{t} \Psi=-i^{2} t \Psi \\
\Rightarrow \hat{t} \Psi=t \Psi \\
\Rightarrow \hat{t}=t
\end{gathered}
$$

This is the Eigen value of time operator.

We know that the commutation relation between time operator and energy operator is " $i \hbar$ " [3].

\section{Commutation Relation between Square of Time Operator and Energy}

Now we will determine the commutation relation between square of time oper- 
ator and energy.

$$
\begin{gathered}
{\left[\hat{t}^{2}, E\right] \Psi=\left(\hat{t}^{2} E-E \hat{t}^{2}\right) \Psi} \\
\Rightarrow\left[\hat{t}^{2}, E\right] \Psi=i \hbar \frac{\partial}{\partial E^{2}}(E \Psi)-i \hbar E \frac{\partial \Psi}{\partial E^{2}} \\
\Rightarrow\left[\hat{t}^{2}, E\right] \Psi=i \hbar\left(\frac{\partial}{\partial E^{2}} E\right) \Psi+i \hbar E \frac{\partial \Psi}{\partial E^{2}}-i \hbar E \frac{\partial \Psi}{\partial E^{2}} \\
\Rightarrow\left[\hat{t}^{2}, E\right] \Psi=0 \\
\Rightarrow\left[\hat{t}^{2}, E\right]=0
\end{gathered}
$$

This is the commutation relation between square of time operator and energy and it is zero. This means, square of time operator and energy commutes.

\section{Wave Functions Which Depends on Position and Time Is Fourier Transform of Wave Function Dependent on Position and Energy}

We can show that two wave functions $\Psi(\boldsymbol{r}, t)$ and $\phi(\boldsymbol{r}, E)$ are Fourier transform of each other. If wave function $\Psi(\boldsymbol{r}, t)$ is given by that,

$$
\begin{gathered}
\Psi(\boldsymbol{r}, t)=A \Psi(\boldsymbol{r}) \mathrm{e}^{-\frac{i E t}{\hbar}} \\
\Rightarrow \Psi_{E}(t)=A \mathrm{e}^{-\frac{i E t}{\hbar}} \\
\Rightarrow\left\langle t \mid \Psi_{E}\right\rangle=A \mathrm{e}^{-\frac{i E t}{\hbar}} \\
\Rightarrow\left\langle\Psi_{E} \mid t^{\prime}\right\rangle=A^{*} \mathrm{e}^{-\frac{i E t^{\prime}}{\hbar}}
\end{gathered}
$$

By multiplying Equations (4) and (5),

$$
\begin{gathered}
\left\langle t \mid \Psi_{E}\right\rangle\left\langle\Psi_{E} \mid t^{\prime}\right\rangle=|A|^{2} \mathrm{e}^{\frac{i E\left(t^{\prime}-t\right)}{\hbar}} \\
\Rightarrow|A|^{2} \int \mathrm{e}^{\frac{i E\left(t^{\prime}-t\right)}{\hbar}} \mathrm{d} E=\int\left\langle t \mid \Psi_{E}\right\rangle\left\langle\Psi_{E} \mid t^{\prime}\right\rangle \mathrm{d} E \\
\Rightarrow|A|^{2} \int \mathrm{e}^{\frac{i E\left(t^{\prime}-t\right)}{\hbar}} \mathrm{d} E=\int\langle t \mid E\rangle\left\langle E \mid t^{\prime}\right\rangle \mathrm{d} E \\
\Rightarrow|A|^{2} \int \mathrm{e}^{\frac{i E\left(t^{\prime}-t\right)}{\hbar}} \mathrm{d} E=\left\langle t \mid \int E\right\rangle\left\langle E \mid t^{\prime}\right\rangle \mathrm{d} E \\
\Rightarrow|A|^{2} \int \mathrm{e}^{\frac{i E\left(t^{\prime}-t\right)}{\hbar}} \mathrm{d} E=\left\langle t \mid t^{\prime}\right\rangle \\
\Rightarrow|A|^{2} \int \mathrm{e}^{\frac{i E\left(t^{\prime}-t\right)}{\hbar}} \mathrm{d} E=\delta\left(t-t^{\prime}\right) \\
\Rightarrow|A|^{2} \int \mathrm{e}^{\frac{i E\left(t^{\prime}-t\right)}{\hbar}} \mathrm{d} E=\frac{1}{2 \pi} \int \mathrm{e}^{\left(t-t^{\prime}\right)} \mathrm{d} \omega \\
\Rightarrow|A|^{2} \int \mathrm{e}^{\frac{i E\left(t^{\prime}-t\right)}{\hbar}} \mathrm{d} E=\frac{1}{2 \pi \hbar} \int \mathrm{e}^{\left(t-t^{\prime}\right)} \mathrm{d}(\hbar \omega)
\end{gathered}
$$


In the left hand side, We interchange $t \& t^{\prime}$ and get

$$
\begin{gathered}
\Rightarrow|A|^{2} \int \mathrm{e}^{\frac{i E\left(t-t^{\prime}\right)}{\hbar}} \mathrm{d} E=\frac{1}{2 \pi \hbar} \int \mathrm{e}^{i E\left(t-t^{\prime}\right)} \mathrm{d} E \\
\Rightarrow|A|^{2}=\frac{1}{2 \pi \hbar} \\
\Rightarrow A=\frac{1}{\sqrt{2 \pi \hbar}}
\end{gathered}
$$

Substituting the value of $A$ into Equation (3),

$$
\begin{aligned}
& \Rightarrow\left\langle t \mid \Psi_{E}\right\rangle=\frac{1}{\sqrt{2 \pi \hbar}} \mathrm{e}^{-\frac{i E t}{\hbar}} \\
& \Rightarrow\langle t \mid E\rangle=\frac{1}{\sqrt{2 \pi \hbar}} \mathrm{e}^{-\frac{i E t}{\hbar}} \\
& \Rightarrow\langle E \mid t\rangle=(\sqrt{2 \pi \hbar})^{-\frac{1}{2}} \mathrm{e}^{\frac{i E t}{\hbar}}
\end{aligned}
$$

Now,

$$
\begin{gathered}
\langle t \mid \alpha\rangle=\int \mathrm{d}^{3} E\langle t \mid E\rangle\langle E \mid \alpha\rangle \\
\Rightarrow\langle t \mid \alpha\rangle=\int \mathrm{d}^{3} E(\sqrt{2 \pi \hbar})^{-\frac{1}{2}} \mathrm{e}^{-\frac{i E t}{\hbar}}\langle E \mid \alpha\rangle \\
\Rightarrow\langle t \mid \alpha\rangle=(\sqrt{2 \pi \hbar})^{-\frac{1}{2}} \int\langle E \mid \alpha\rangle \mathrm{e}^{-\frac{i E t}{\hbar}} \mathrm{d}^{3} E \\
\Rightarrow\left\langle t \mid \Psi_{\alpha}\right\rangle=(\sqrt{2 \pi \hbar})^{-\frac{1}{2}} \int\left\langle E \mid \varphi_{\alpha}\right\rangle \mathrm{e}^{-\frac{i E t}{\hbar}} \mathrm{d}^{3} E \\
\Rightarrow \Psi_{\alpha}(t)=(\sqrt{2 \pi \hbar})^{-\frac{1}{2}} \int \varphi_{\alpha}(E) \mathrm{e}^{-\frac{i E t}{\hbar}} \mathrm{d}^{3} E
\end{gathered}
$$

Again,

$$
\begin{gathered}
\langle E \mid \alpha\rangle=\int \mathrm{d}^{3} t\langle E \mid t\rangle\langle t \mid \alpha\rangle \\
\Rightarrow\langle E \mid \alpha\rangle=(\sqrt{2 \pi \hbar})^{-\frac{1}{2}} \int \mathrm{e}^{\frac{i E t}{\hbar}} \mathrm{d}^{3} t\langle t \mid \alpha\rangle \\
\Rightarrow\langle E \mid \alpha\rangle=(\sqrt{2 \pi \hbar})^{-\frac{1}{2}}\langle t \mid \alpha\rangle \int \mathrm{e}^{\frac{i E t}{\hbar}} \mathrm{d}^{3} t \\
\Rightarrow\left\langle E \mid \varphi_{\alpha}\right\rangle=(\sqrt{2 \pi \hbar})^{-\frac{1}{2}}\left\langle t \mid \Psi_{\alpha}\right\rangle \int \mathrm{e}^{\frac{i E t}{\hbar}} \mathrm{d}^{3} t \\
\Rightarrow \varphi_{\alpha}(E)=(\sqrt{2 \pi \hbar})^{-\frac{1}{2}} \Psi_{\alpha}(t) \int \mathrm{e}^{\frac{i E t}{\hbar}} \mathrm{d}^{3} t
\end{gathered}
$$

We can generalize (8) \& (9) respectively and write these equations,

$$
\begin{gathered}
\Psi(t)=(\sqrt{2 \pi \hbar})^{-\frac{1}{2}} \int \varphi(E) \mathrm{e}^{-\frac{i E t}{\hbar}} \mathrm{d}^{3} E \\
\varphi(E)=(\sqrt{2 \pi \hbar})^{-\frac{1}{2}} \int \Psi(t) \mathrm{e}^{\frac{i E t}{\hbar}} \mathrm{d}^{3} t
\end{gathered}
$$


We can rewrite Equation (3) into this form,

$$
\begin{gathered}
\Psi(\boldsymbol{r}, t)=\Psi(t) \mathrm{e}^{i \frac{\boldsymbol{p} \cdot \boldsymbol{r}}{\hbar}} \\
\Rightarrow \Psi(\boldsymbol{r}, t)=\mathrm{e}^{i \frac{\boldsymbol{p} \cdot \boldsymbol{r}}{\hbar}}(\sqrt{2 \pi \hbar})^{-\frac{1}{2}} \int \varphi(E) \mathrm{e}^{-\frac{i E t}{\hbar}} \mathrm{d}^{3} E \\
\Rightarrow \Psi(\boldsymbol{r}, t)=(\sqrt{2 \pi \hbar})^{-\frac{1}{2}} \int \varphi(E) \mathrm{e}^{i \frac{\boldsymbol{p} \cdot \boldsymbol{r}}{\hbar}} \mathrm{e}^{-\frac{i E t}{\hbar}} \mathrm{d}^{3} E \\
\Rightarrow \Psi(\boldsymbol{r}, t)=(\sqrt{2 \pi \hbar})^{-\frac{1}{2}} \int \varphi(E, \boldsymbol{r}) \mathrm{e}^{-\frac{i E t}{\hbar}} \mathrm{d}^{3} E
\end{gathered}
$$

where, $\varphi(E, \boldsymbol{r})=\varphi(E) \mathrm{e}^{i \frac{\boldsymbol{p} \cdot \boldsymbol{r}}{\hbar}}$.

Like Equation (12) we can write energy dependent wave function like this,

$$
\begin{gathered}
\varphi(\boldsymbol{r}, E)=\varphi(E) \mathrm{e}^{i \frac{\boldsymbol{p} \cdot \boldsymbol{r}}{\hbar}} \\
\Rightarrow \varphi(\boldsymbol{r}, E)=(\sqrt{2 \pi \hbar})^{-\frac{1}{2}} \int \Psi(t) \mathrm{e}^{\frac{i E t}{\hbar}} \mathrm{d}^{3} t \mathrm{e}^{i \frac{\boldsymbol{p} \cdot \boldsymbol{r}}{\hbar}} \\
\Rightarrow \varphi(\boldsymbol{r}, E)=(\sqrt{2 \pi \hbar})^{-\frac{1}{2}} \int \Psi(t) \mathrm{e}^{i \frac{\boldsymbol{p} \cdot \boldsymbol{r}}{\hbar}} \mathrm{e}^{\frac{i E t}{\hbar}} \mathrm{d}^{3} t \\
\Rightarrow \varphi(\boldsymbol{r}, E)=(\sqrt{2 \pi \hbar})^{-\frac{1}{2}} \int \Psi(\boldsymbol{r}, t) \mathrm{e}^{\frac{i E t}{\hbar}} \mathrm{d}^{3} t
\end{gathered}
$$

where, $\Psi(\boldsymbol{r}, t)=\Psi(t) \mathrm{e}^{i \frac{\boldsymbol{p} \cdot \boldsymbol{r}}{\hbar}}$.

From Equations (13) \& (14) we can see that wave function.

$\Psi(\boldsymbol{r}, t)$ and $\varphi(\boldsymbol{r}, E)$ are each other's Fourier transform. Until now, wave functions only depend on position, time, and momentum. For the first time, I have showed that wave also depend on energy.

\section{Expectation Value of Time Operator}

Now we will see that the expectation value of time operator is real.

$$
\begin{gathered}
\langle t\rangle=\int_{-\infty}^{\infty} \Psi^{*} t \Psi \mathrm{d} E \\
\Rightarrow\langle t\rangle=i \hbar \int_{-\infty}^{\infty} \Psi^{*} \frac{\partial}{\partial E} \Psi \mathrm{d} E \\
\Rightarrow\langle t\rangle=i \hbar\left[\Psi^{*} \int \frac{\partial \Psi}{\partial E} \mathrm{~d} E\right]_{-\infty}^{\infty}-i \hbar \int_{-\infty}^{\infty}\left(\frac{\partial \Psi^{*}}{\partial E} \int \frac{\partial \Psi}{\partial E} \mathrm{~d} E\right) \mathrm{d} E \\
\Rightarrow\langle t\rangle=0-i \hbar \int_{-\infty}^{\infty}\left(\frac{\partial \Psi^{*}}{\partial E} \Psi\right) \mathrm{d} E \\
\Rightarrow\langle t\rangle=\int_{-\infty}^{\infty} \Psi\left(i \hbar \frac{\partial \Psi^{*}}{\partial E}\right)^{*} \mathrm{~d} E \\
\Rightarrow\langle t\rangle=\int_{-\infty}^{\infty} \Psi t \Psi^{*} \mathrm{~d} E \\
\Rightarrow\langle t\rangle=\int_{-\infty}^{\infty}\left(\Psi^{*} t \Psi\right)^{*} \mathrm{~d} E
\end{gathered}
$$




$$
\Rightarrow\langle t\rangle=\langle t\rangle^{*}
$$

This is possible only when time operator's expectation value is real number. So $\langle t\rangle$ is real. In other papers [2] [3], it has proved that time operator is a Hermitian operator. If any operator is Hermitian then its expected value is real. I don't prove that time operator's self-adjointness but directly prove that time operator's expectation value is real.

\section{Conclusion}

Definition of time operator and its commutation relation with energy are given in many papers [2] [3], but we have shown that its square commutes with energy; $\Psi(\boldsymbol{r}, t)$ and $\varphi(\boldsymbol{r}, E)$ are each other's Fourier transform and expectation value of time operator is real. We also have determined its Eigen value. Readers of this paper can use time operator and create a new kind of equation of motion which will become alternate of Schrodinger equation.

\section{Conflicts of Interest}

The author declares no conflicts of interest regarding the publication of this paper.

\section{References}

[1] Zettili, N. (2003) Quantum Mechanics: Concepts and Applications. American Journal of Physics, 71, 93. https://doi.org/10.1119/1.1522702

[2] Wang, Z.-Y. and Xiong, C.-D. (2007) How to Introduce Time Operator. Annals of Physics, 322, 2304-2314. https://doi.org/10.1016/j.aop.2006.10.007

[3] Olkhovsky, V., Recami, E. and Gerasimchuk, A. (1974) Time Operator in Quantum Mechanics. Il Nuovo Cimento A (1965-1970), 22, 263-278.

https://doi.org/10.1007/BF02813438 